\title{
Microwave reference signal distribution on optical fibre within satellite payload
}

K. Balakier, V. M. Fernandez Laguna, R. Green

K. Balakier, V. M. Fernandez Laguna, R. Green, "Microwave reference signal distribution on optical fibre within satellite payload," Proc. SPIE 11852, International Conference on Space Optics - ICSO 2020, 1185252 (11 June 2021); doi: 10.1117/12.2602916

SPIE Event: International Conference on Space Optics - ICSO 2021, 2021, Online Only 


\section{International Conference on Space Optics-ICSO 2020}

Virtual Conference

30 March-2 April 2021

Edited by Bruno Cugny, Zoran Sodnik, and Nikos Karafolas
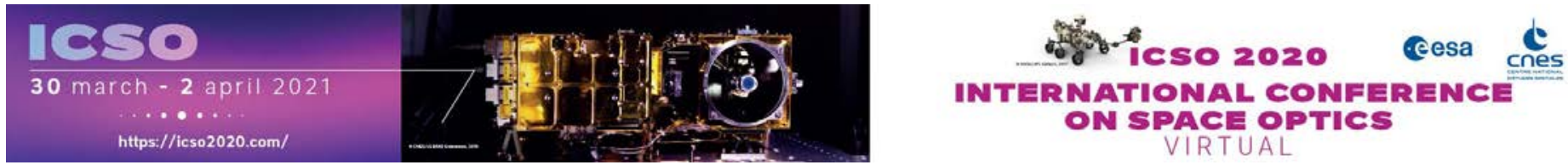

\section{Microwave reference signal distribution on optical fibre within satellite payload}

\section{Cesa iso poreceatings ecres}




\title{
Microwave reference signal distribution on optical fibre within satellite payload
}

\author{
K. Balakier, V. M. Fernandez Laguna, R. Green \\ Airbus Defence and Space, Gunnels Wood Road, Stevenage SG1 2AS, UK
}

\begin{abstract}
This paper will discuss mission requirements and possible architectures of optical fibre network for the distribution of Master Local Oscillator (MLO) signals to multiple RF input ports on-board Satellite payloads. With that purpose, each of the MLO signals is modulated onto a different CW optical carrier in order to be distributed and power is equally split into multiple fibre paths while in the optical domain. The MLO signals to be delivered can be either lower power digital signals as well as high power analogue signals $(>0 \mathrm{dBm})$, and/or a combination of both, in the frequency range between $1 \mathrm{GHz}$ and $20 \mathrm{GHz}$.

The novel contribution of this paper is the comparison of two architectures whose main distinction is a different number of amplifiers against the required RF ports. This in turn defines the optimum way for optical amplification implementation in the optical signal distribution network. The trade-off shows that for the high number of MLOs and smaller number $(<32)$ of RF output ports the wavelength division multiplexing (WDM) before optical amplification is preferred, while for the low MLO number and high number $(>100)$ of RF ports distribution network the high power highly efficient optical power amplification is more beneficial. These two types of networks are compared focusing on the DC power consumption of all the equipment and the flexibility to accommodate them onto the Spacecraft.
\end{abstract}

Keywords: RF over fibre, signal distribution network, vHTS, master local oscillator, microwave photonics

\section{INTRODUCTION}

The large geostationary very high throughput communication satellites (VHTS) contain user antennas consisting of a large number of elements required to generate many beams, which need to be steerable. The large number of antenna elements drives a number of ADCs and DACs, which are needed by the on-board-processor to process the signal received by antenna array before it is transmitted back to the ground. This large number of elements creates a connectivity challenge, not only for the received/transmit signal paths but also for RF reference signal distribution needed by the ADCs and DACs. This paper considers and compares different architectures for the RF master local oscillator (MLO) signal distribution from the centralised frequency generation unit (FGU) where the high purity MLO signal is generated to multiple ADCs. Such MLO signal could be distributed using RF cables, however the large number of elements makes the use of RF cables very questionable, due to i) cable mass, ii) complexity of cables distribution within the payload iii) electromagnetic interference between the cables, and iv) cable propagation loss which becomes even more prominent over longer cable runs and at the higher frequencies ( $\mathrm{Ku}$ - and $\mathrm{Ka}-\mathrm{band})$. To mitigate these issues, the use of optical fibres to transport RF signals, e.g. RF over fibre (RFoF), has been considered. Precisely, the use of RFoF links has continued to expand over the last decades mainly due to terrestrial antenna remoting applications [1], [2]. More recently, it has been also considered for intra-satellite applications, including reference signal distribution [3]. Transporting MLO signal on an optical fibre network can have distinct advantages, including thanks to low weight of optical fibre and the excellent isolation between the fibre links. However, the process of electrical-optical-electrical conversion is inefficient and multiple split introduces further reduction in signal power. Therefore signal distribution network based on RF-optical-RF links should be designed with little negative gain using a suitable optical amplifier and $\mathrm{RF}$ post amplification. Proposed system is designed to carry MLO frequencies from 1.5 to $20 \mathrm{GHz}$ along with digital signals modulated over individual optical fibres and then splitting the signals. This contrasts with most RFoF links, 
where only point to point connectivity is provided. In the case of MLO signal distribution the same signal is often required to be delivered to multiple points, which requires the signal to be split inside the FGU and distributed on multiple parallel RF cables. This solution, although perfectly functional, creates a challenge when the number of ports which require MLO signal increases to a few hundreds. This paper investigates an alternative solution where the high purity signal, although still generated at FGU would be modulated on to an optical carrier, split multiple ways and subsequently distributed on optical fibre.

\section{MASTER LOCAL OSCILLATOR SIGNAL}

The FGU is designed to generate a range of different frequencies, which are the harmonics of Master Reference Oscillator (MRO), which is implemented with at least 2 for 1 redundancy. The exact frequency range of the generated MLO signals depends on the uplink (e.g. for downconversion at the ADCs) and downlink (e.g. for upconversion at the DACs) frequencies used by the vHTS mission. A typical FGU is designed to generate several different frequencies, which can fall into a range of frequencies in $\mathrm{S} / \mathrm{C} / \mathrm{X} / \mathrm{Ku} / \mathrm{Ka} / \mathrm{Q} / \mathrm{V}$ bands, plus the sampling frequencies (Fs) for the ADCs and DACs. Each MLO signal is generated by a separate circuit block, which will have as well a 2 for 1 redundancy internally, then split multiple ways for distribution, as shown on Figure 1.

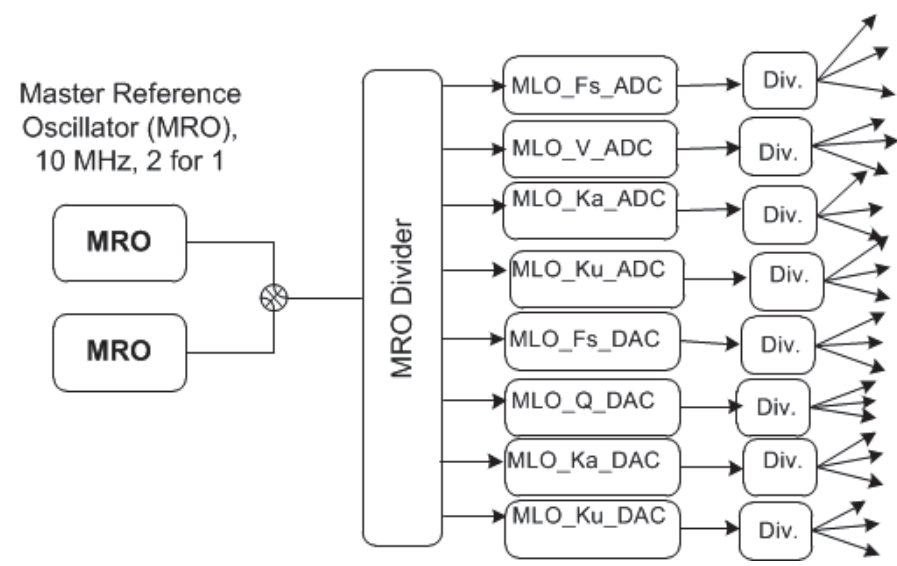

Figure 1 Example of an MLO distribution for a mission operating in frequency bands $\mathrm{Ku}, \mathrm{Ka}, \mathrm{V}$ and $\mathrm{Q}$, plus the sampling frequencies Fs for the ADCs and DACs.

The high purity of the MLO signal is generated at the FGU with phase noise below $-100 \mathrm{dBc} / \mathrm{Hz}$ at $100 \mathrm{kHz}$ frequency offset from the centre frequency and spurious and harmonics below $-50 \mathrm{dBc}$. It is required that the signal is distributed to multiple points without any degradation in the signal purity. The frequency stability is expected to be better than $0.3 \mathrm{ppm}$.

Table 1 Typical characteristics of the MLO signal

\begin{tabular}{|c|l|c|c|}
\hline \multirow{4}{*}{$\begin{array}{c}\text { Phase noise }[\mathrm{dBc} / \mathrm{Hz}] \\
\text { frequency offset of }\end{array}$} & $100 \mathrm{~Hz}$ & -77 & \\
\cline { 2 - 3 } & $1 \mathrm{kHz}$ & -96 \\
\cline { 2 - 3 } & $10 \mathrm{kHz}$ & -96 \\
\cline { 2 - 3 } & $100 \mathrm{kHz}$ & -100 & \multirow{4}{*}{ Typical @ 12 $\mathrm{GHz}$} \\
\cline { 2 - 3 } & $1 \mathrm{MHz}$ & -126 \\
\cline { 2 - 3 } & $10 \mathrm{MHz}$ & -145 & \\
\hline \multicolumn{2}{|c|}{ Spurious and Harmonics } & $\begin{array}{c}\text { Discrete - } \\
50 \mathrm{dBc}\end{array}$ & Harmonic -50dBc \\
\hline
\end{tabular}




\section{SIGNAL DIVISION AND DISTRIBUTION 1 x N}

The outputs of the MLO generating blocks in Figure 1 are implemented with passive RF cables and RF splitters. This could be replaced by an RF-over-fibre (RFoF) link network to reduce the mass, improve isolation between channels and allow for a smaller bending radius of the transmission lines. The considered number of replicas of MLO signals can range between two and 32. To compensate for the power drop due to multiple fibre slitters, a high power erbium doped fibre amplifier (EDFA) would be implemented after the optical modulator and before the optical slitting network, as shown in Figure 2.

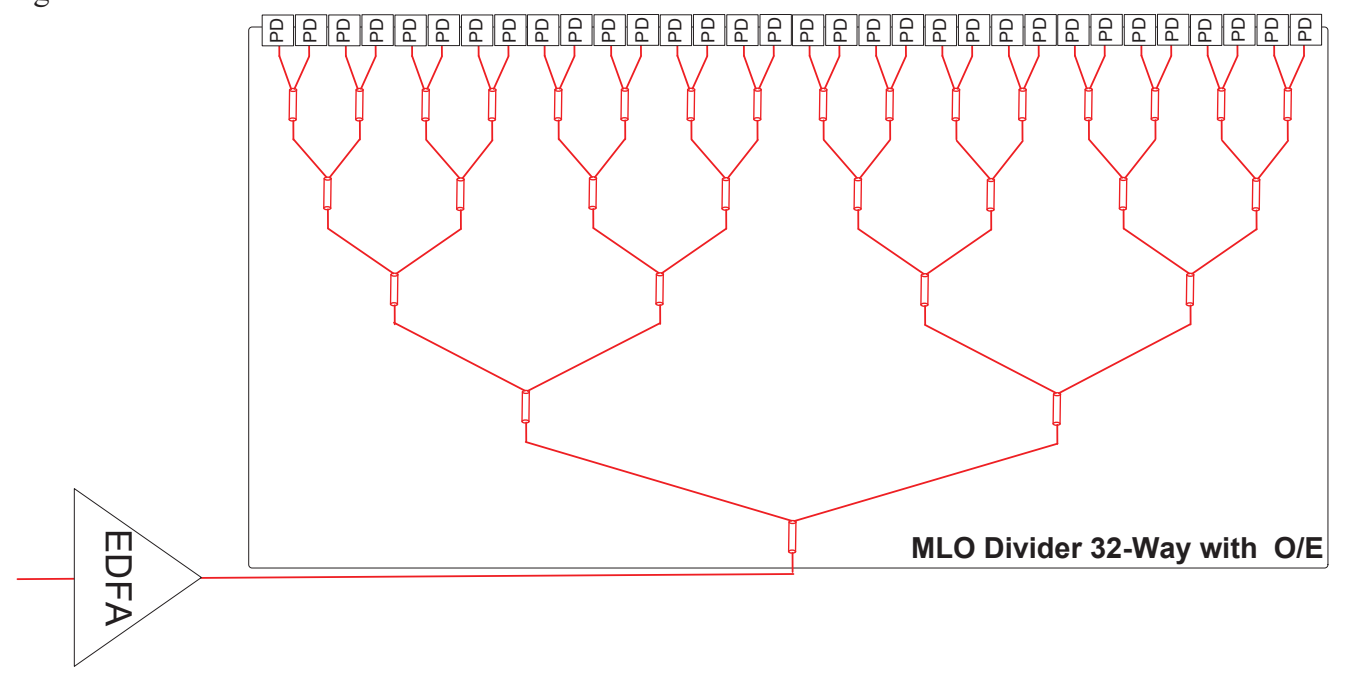

Figure 2 Schematic of 32-way optical splitting network

A range of different EDFAs with different gain, noise figure and output power levels have been considered. Figure 3 represents the corresponding RF power level at the output of each photodiode (PD), for different splitting ratios and different EDFA output power. The calculation assumed a PD responsivity of $0.8 \mathrm{~A} / \mathrm{W}$ and $3 \mathrm{~dB}$ loss per each optical splitter. No additional loss due to the connectivity in the optical path has been considered.

As the RF output power decreases by $2 \mathrm{~dB}$ for every $1 \mathrm{~dB}$ reduction in optical power, it can be noticed that the expected RF power at the photodiodes output can drop significantly with the higher number of optical splitters. For very high splitting ratios, the use of low noise RF amplification after the PD can be considered to meet the required RF power level, while maintaining low phase noise.

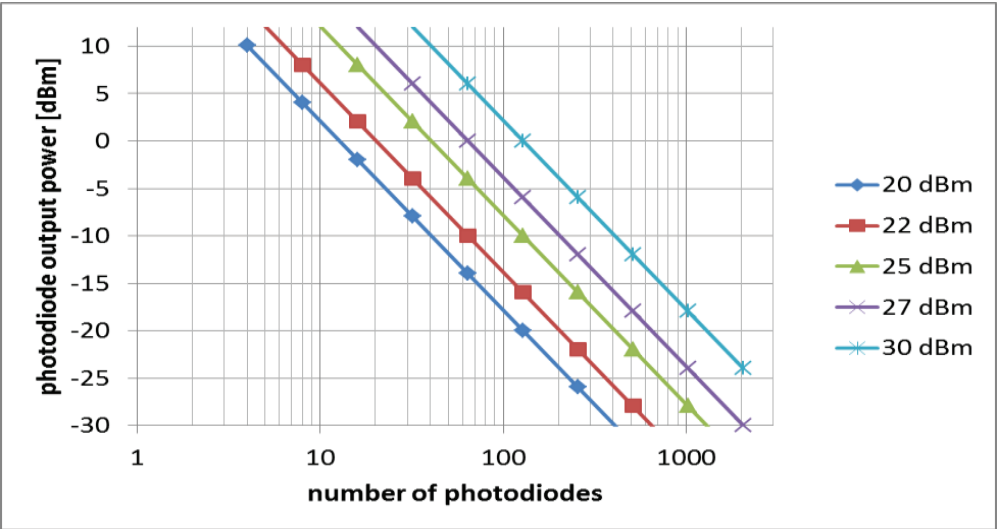

Figure 3 Expected RF power at the output of each photodiode in function of the number of photodiodes and the optical power at the input of the optical splitting network. 
Depending on the payload architecture, some signals may need to be split only one time (one splitter) to be distributed to two ports. This means the use of high power EDFA may be excessive. However, the optical power drop due to the splitters still needs to be compensated. The solution considered for such cases would be for a few MLO signals (modulated on separate optical carriers) to be multiplexed and amplified together on a single EDFA.

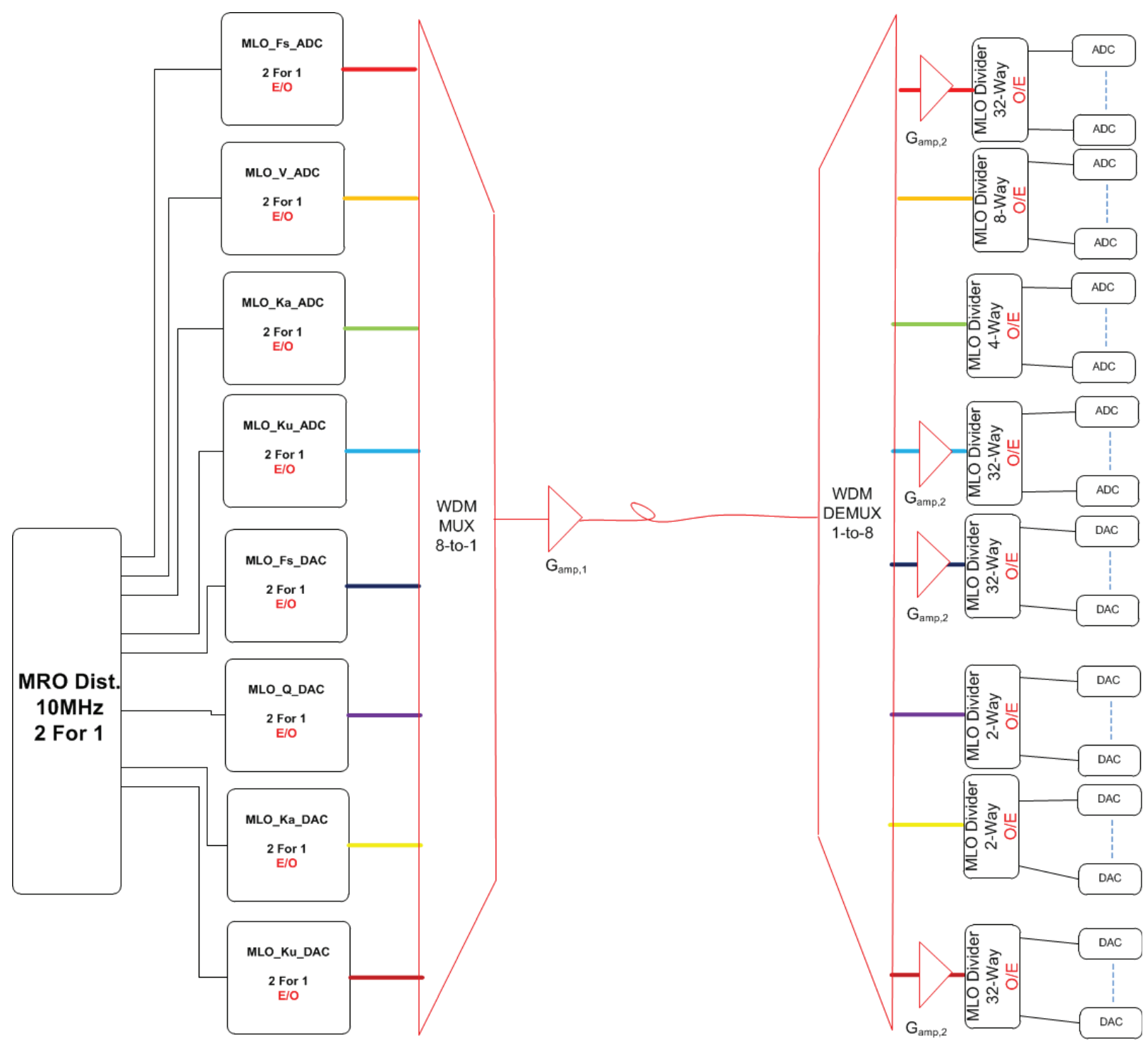

Figure 4 Overall architecture 1, showing channels to be amplified together and other channels having one EDFA per single MLO frequency.

The architecture above is compared with an alternative one in which no WDM is considered, therefore incrementing the number of amplifiers but at the same time reducing the gain and output power requirements for each EDFA: 


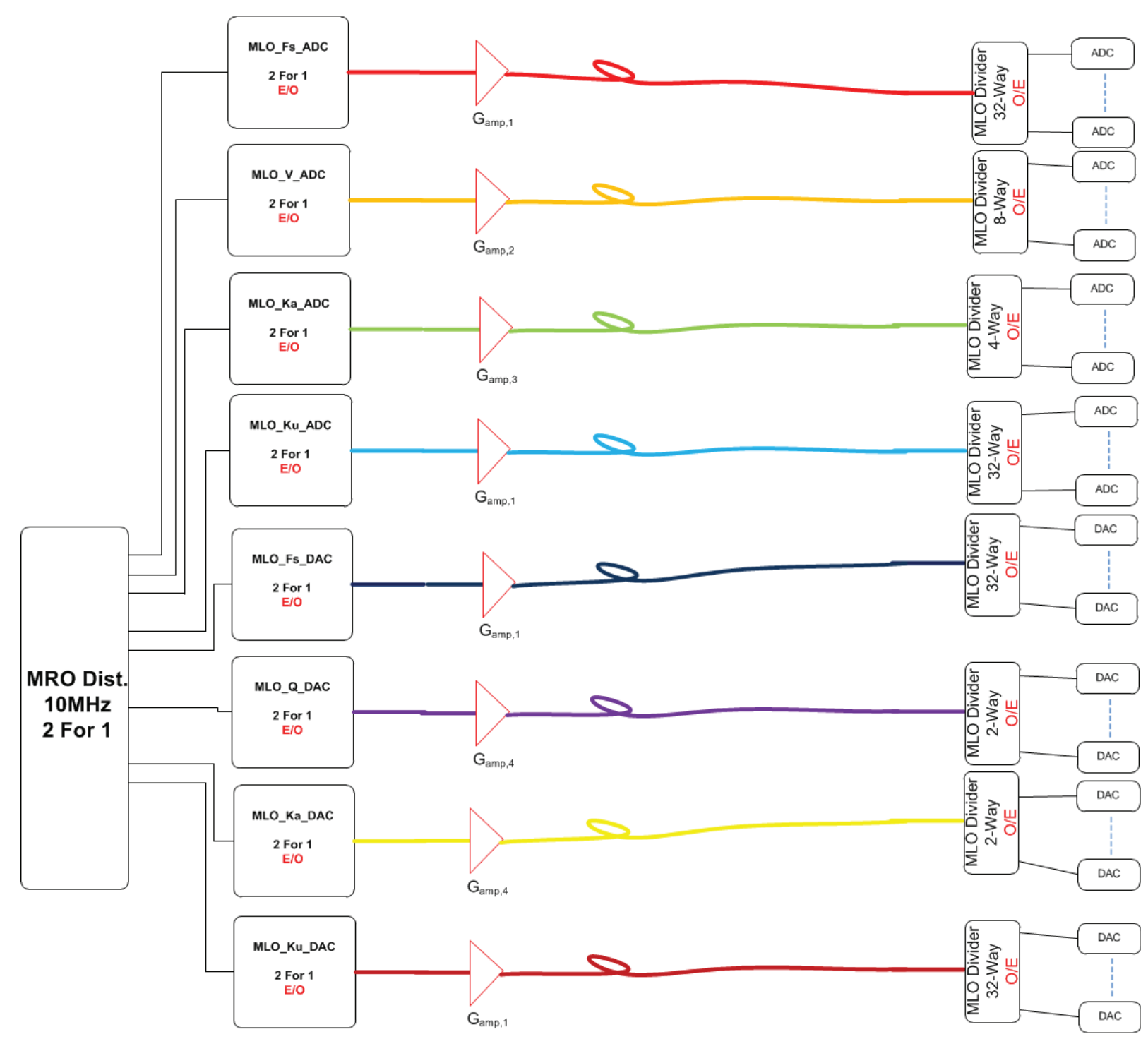

Figure 5 Architecture 2, showing one EDFA per single MLO frequency, e.g. there is no WDM and consequently no EDFA sharing.

The implementation of EDFAs introduces additive optical noise, mainly due to amplified spontaneous emission (ASE) into the optical network. This may lead to the reduction of signal-to-noise ratio. However, the distributed MLO signal is a high power tone at single frequency, which can be well recovered after the PD. The extremely low noise figure of the EDFA, as well as its exact location in front of the splitting network, has been considered in the system design. The phase noise measurement of MLO signal at $20 \mathrm{GHz}$ frequency distributed on the RF cable has been compared with the measurement of the signal distributed on fibre network, demonstrating no phase noise degradation due to the amplified optical fibre network. 


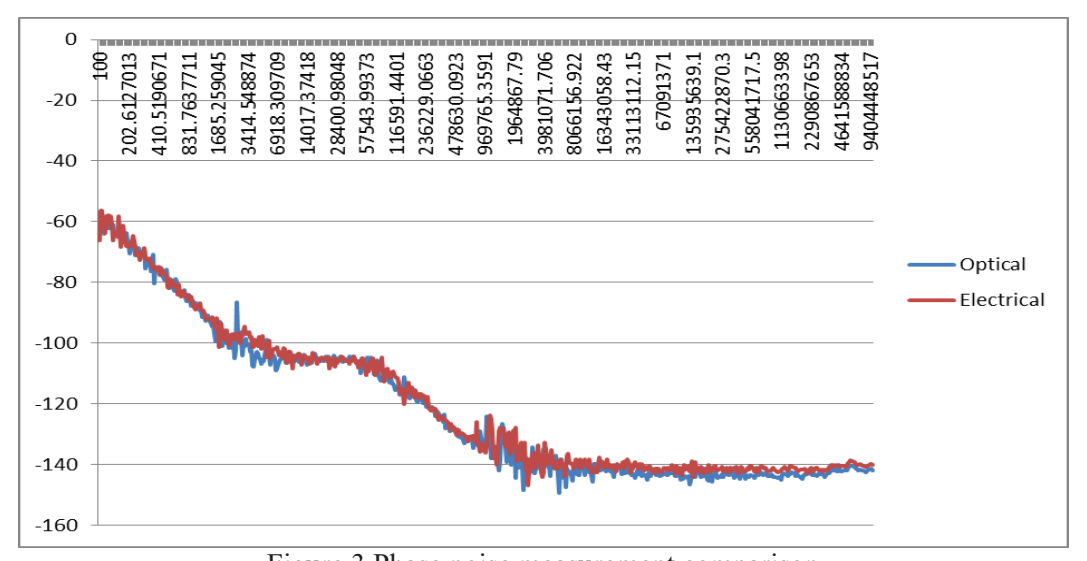

Figure 3 Phase noise measurement comparison

\section{POWER CONSUMPTION AND ACCOMODATION FLEXIBILITY}

The overall DC power consumption of the optical network is an important factor when considering optical technology for the MLO signal distribution. The main contributors to the total power consumption are EDFAs. Depending on the splitting ratio of each individual signal, the range of EDFAs with output power between $125 \mathrm{~mW}$ and $2 \mathrm{~W}$ has been considered. Figures 4 and 5 represent two architectures of optical distribution networks. Although the number of MLO input signals and corresponding photodiode outputs (101 in total) is the same in both cases, the total DC power corresponding to architecture 1 (Figure 4) is $8 \mathrm{~W}$ higher (totalling to $250 \mathrm{~W}$ ) than the power of architecture 2 (figure 5) which has a total value of $242 \mathrm{~W}$. However, the number of EDFAs in architecture 2 is higher than in architecture 1 , which will have an impact on the total mass of the optical distribution network, particularly when the redundancy amplifier units are taken into consideration.

From the perspective of flexibility of accommodation of optical equipment within the spacecraft, the use of low loss optical fibre offers strong advantage, as it allows for the remote location of the FGU. After comparing both architectures, it has become evident that architecture 1 with WDM offers a better trade-off for the VHTS payloads with the large number of ports, as it requires a smaller number of EDFA units but introduces some constraints into the signal guiding path due to WDM. Architecture 2 allows for a different disaggregation of the EDFA units, which is much more convenient for smaller payloads with a lower number of ports which require MLO signal. Architecture 2 also offers full flexibility in the location of EDFAs and optical harness.

\section{CONCLUSIONS}

It is estimated that the efficiency of RF amplifiers used in the traditional MLO distribution network is superior to the efficiency of optical amplifiers; however, the low loss of the optical fibre compared to the RF cables represents a significant advantage at the system level. The decision whether an MLO distribution network should be all-electrical or, on the contrary, use fibre distribution technologies, will ultimately depend on the number of distribution ports and the frequency range of the local oscillators to distribute.

When the total number of distribution ports is small and the distribution frequencies are below $20 \mathrm{GHz}$, an all-electrical distribution network seems most appropriate.

When the number of distribution ports increases to the order of hundreds, the lightweight of optical fibres becomes a winning factor. At the same time, when the LO frequencies are higher than $20 \mathrm{GHz}$, optical distribution can outperform the electrical. The consequence of optical distribution is the need to use optical amplifiers to compensate for the signal 
split and electro-optical and opto-electrical conversion losses. For this reason, different types of architectures have been compared and looked at from the power dissipation and equipment accommodation point of views. The analyses have been carried out taking into consideration the number of distribution ports and required RF power level at each port. In this paper, we compared two optical distribution architectures against the same scenario (100+ photodiode output ports), and it was concluded that the use of WDM followed by amplification is a preferred MLO distribution architecture due to small power consumption difference and a smaller volume of the required equipment. However, optical amplification without a WDM would be preferable, should there be a smaller number of LO frequencies and high number of output ports.

\section{ACKNOWLEDGEMENT}

This work was performed within the Airbus Defence and Space and ESA ARTES Scylight contract number 4000129688 /20/NL/AF

\section{REFERENCES}

[1] D. Novak et al., "Radio-Over-Fiber Technologies for Emerging Wireless Systems," in IEEE Journal of Quantum Electronics, vol. 52, no. 1, pp. 1-11, Jan. 2016, Art no. 0600311

[2] E. Ackerman, C. Cox, J. Dreher, M. Davis, and D. DeBoer, "Fiber-Optic Antenna Remoting for Radioastronomy Applications," URSI 27th General Assembly, Maastricht, 2002.

[3] Miguel A. Piqueras, Javier Martí, Silvia Delgado, Rob Singh, Robert Parish, Ghislain Turgeon, Agustin Zornoza, "A Ka-band single string photonic payload flight demonstrator for broadband high throughput satellite systems and an in orbit demonstrator of optical RF distribution on board satellites," Proc. SPIE 11180, International Conference on Space Optics — ICSO 2018, 111807F (12 July 2019); 